\title{
A NOTE ON COMPLETE SEPARATION IN THE STONE TOPOLOGY
}

\author{
G. A. JENSEN
}

Let $L$ be the lattice of all ideals of a ring with unity and let $X$ be a subset of the set of all real ideals of $A$ [1] which is equipped with the Stone topology [4, p. 272]. Anderson [1, Lemma 4, 5(2)] gave necessary and sufficient conditions, in terms of certain elements of $L$, for two subsets of $X$ to be completely separated in $X$. In [6, §8], we modified the idea of Anderson in case $L$ is the lattice of all $S_{\alpha}$-ideals of a $C$-lattice $[2, \S \S 2$ and 3] for $\alpha$ a real number and $X$ a certain subset of $L$ equipped with the Stone topology. The purpose of this note is to give necessary and sufficient conditions for two subsets of $X$ to be completely separated in $X$ in case $L$ is an arbitrary complete lattice and $X$ is a subset of $L$ which is completely regular when equipped with the Stone topology. The idea is essentially that of Anderson in [1]. We use this result to complete the "internal" characterization of the $\Phi$-algebra of all real-valued continuous functions on an arbitrary completely regular space for which Henriksen and Johnson [6] have results in special cases.

Suppose now that $L$ is a complete lattice and $X$ is a fixed subset of $L$. We define a function $\phi$ from $L$ into $L$ by

$$
\phi(x)=\wedge\{y \in X \mid x \leqq y\} \quad(x \in L)
$$

and we let $K=\{x \in L \mid x=\phi(x)\}$. It is clear that $\phi(x)$ is merely the meet of the intersection of $X$ with the principal dual ideal of $L$ generated by $x$. It is also a routine matter to verify that $\phi$ is a closure operation [3, p. 49] on $L$ and hence by [8, Theorem 4.1], $K$ is a complete lattice relative to the partial order on $L$. Moreover, meets in $K$ coincide with meets in $L$ and joins in $K$ are obtained by operating on the joins in $L$ by $\phi$. In addition, $X \subset K, \wedge X=\wedge K$, and if $1=\vee L$, then $1 \in K$.

We now suppose that $X$ is also equipped with the Stone topology. Using the fact that the closure of a subset $Y$ of $X$ is $\{y \in X \mid \wedge Y \leqq y\}$, one can show that the mapping $F \rightarrow \Lambda F$ is a dual isomorphism of the lattice of all closed subsets of $X$ onto $K$.

Next, we define a binary relation $*$ on $K$ as follows: $x * y$ in case

Presented to the Society, January 27, 1966 under the title Complete separation in the Stone topology; received by the editors October 12, 1966 and, in revised form, April 1, 1968. 
there exists $z \in K$ such that $z \wedge x=\Lambda X$ and $z \vee y=1$. The motivation for this definition is the following: If $F_{1}$ and $F_{2}$ are closed subsets of $X$, then $F_{2} \subset$ int $F_{1}$ if and only if $\Lambda F_{1} * \Lambda F_{2}$. Note that this implies that $*$ is a transitive relation on $K$.

If $C \subset K$, we say that $C$ is a dense chain with respect to $*$ in Case (i) for each $x, y \in C$, either $x * y$ or $y * x$, and (ii) if $x, y \in C$ with $x * y$, then there exists $z \in C$ such that $x * z * y$. We now define a binary relation $\perp$ on $K$ by $x \perp y$ in case there exists a countable subset $C$ of $K$ such that $C$ is a dense chain with respect to $*, x \leqq \Lambda C$, and $\vee C \leqq y$.

Theorem 1. Let $X$ be a subset of a complete lattice $L$ so that $X$ is a completely regular space when equipped with the Stone topology. Subsets $A_{1}$ and $A_{2}$ of $X$ are completely separated in $X$ if and only if there exist closed subsets $F_{1}$ and $F_{2}$ of $X$ such that $A_{1} \subset X \backslash F_{1}, A_{2} \subset F_{2}$, and $\bigwedge F_{1} \perp \Lambda F_{2}$.

Proof. Let $Q$ denote the rational numbers. It is not difficult to show that subsets $A_{1}$ and $A_{2}$ of a completely regular space $X$ are completely separated in $X$ if and only if there exist closed sets $F_{1}$ and $F_{2}$ of $X$ and a family $\left(U_{t}\right)_{t \in Q}$ of open sets of $X$ such that $A_{1} \subset X \backslash F_{1} \subset \cap_{t \in Q} U_{t}, \cup_{t \in Q} U_{t} \subset X \backslash F_{2} \subset X \backslash A_{2}$, and $r<s$ in $Q$ implies that $\operatorname{cl} U_{r} \subset U_{s}$.

Suppose first that $A_{1}$ and $A_{2}$ are completely separated in $X$, and let $F_{1}, F_{2}$, and $\left(U_{t}\right)_{t \in Q}$ be as described above. For each $t \in Q$, let $F_{t}=X \backslash U_{t}$. One can show that $\left\{\Lambda F_{t} \mid t \in Q\right\}$ is a countable dense chain with respect to $*$ such that $\Lambda F_{1} \leqq \Lambda\left\{\Lambda F_{t} \mid t \in Q\right\}$ and $\bigvee\left\{\Lambda F_{t} \mid t \in Q\right\}$ $\leqq \bigwedge F_{2}$.

Conversely, if there exist closed sets $F_{1}$ and $F_{2}$ of $X$ such that $A_{1} \subset X \backslash F_{1}, A_{2} \subset F_{2}$, and $\Lambda F_{1} \perp \wedge F_{2}$, then there exists a countable subset $C$ of $K$ such that $C$ is a dense chain with respect to $*, \Lambda F_{1} \leqq \Lambda C$, and $\bigvee C \leqq \Lambda F_{2}$. The conditions on $C$ imply that there exists a function $f: Q \rightarrow C$ such that $r<s$ in $Q$ implies that $f(r) * f(s)$. For each $t \in Q$, let $U_{t}$ be the open subset of $X$ defined by $\Lambda\left(X \backslash U_{t}\right)=f(t)$. It is a routine matter to verify that $\left(U_{t}\right)_{t \in Q}$ is a family of open sets satisfying $X \backslash F_{1} \subset \bigcap_{t \in Q}, \cup_{t \in Q} U_{t} \subset X \backslash F_{2}$, and $r<s$ in $Q$ implies that cl $U_{r} \subset U_{s}$. Hence the theorem.

Suppose now that $A$ is a $\Phi$-algebra. We refer the reader to [5] for definitions, symbols, and notation not defined here. Let $L$ be the complete lattice of all $l$-ideals of $A$, let $\Omega(A)$ be the set of all real maximal $l$-ideals of $A$ equipped with the Stone topology, and let $K(A)$ be the subset of $L$ as defined earlier using the operator $\phi$ and the subset $R(A)$ of $L$. It follows that $I \in K(A)$ if and only if 
$I=\bigcap\{M \in R(A) \mid I \subset M\}$. If $\cap \mathbb{R}(A)=\{0\}$, the binary relation $*$ can be restated slightly as follows: $I_{1} * I_{2}$ in case there exists $J \in K$ with $I_{1} \cap J=\{0\}$ and $I_{2} \cup J \nsubseteq M$ for any $M \in R(A)$.

We can now obtain an "internal" characterization as a $\Phi$-algebra of the set $C(X)$ of all real-valued continuous functions on an arbitrary completely regular space $X$.

Theorem 2 (Compare $[5,5.2]$ ). $A$-algebra $A$ is isomorphic to $C(X)$ for some completely regular space $X$ if and only if

(i) $A$ is an algebra of real-valued functions,

(ii) $A$ is uniformly closed,

(iii) $A$ is closed under inversion, and

(iv) for each pair $I \perp J$ in $K(A)$, there exists $f \in A$ such that $f-1 \in J$ and $f \wedge|h|=0$ for all $h \in I$.

PRoof. The only condition which needs comment concerning the necessity of the conditions is (iv). It follows easily from 4.6 in [5] and the fact that $I \perp J$ in $K(A)$ if and only if the corresponding sets in $\mathcal{R}(A)$ are completely separated.

In view of the proof of 5.2 in [5], the sufficiency of the four conditions will be immediate if it is shown that disjoint zero-sets in $\mathbb{R}(A)$ have disjoint closures in $\mathfrak{N}(A)$, where $\mathfrak{T}(A)$ is the space of all maximal $l$-ideals of $A$. Let $Z_{1}$ and $Z_{2}$ be disjoint zero-sets in $R(A)$. Then there exist closed subsets $F_{1}$ and $F_{2}$ in $R(A)$ such that $Z_{1} \subset Q(A) \backslash F_{1}$, $Z_{2} \subset F_{2}$, and $\cap F_{1} \perp \cap F_{2}$. By (iv), there exists $f \in A$ such that $f-1 \in \cap F_{2}$ and $f \wedge|h|=0$ for all $h \in \cap F_{1}$. Thus, if $M \in Z_{1}$, then $M \in F_{1}$ and so $\cap F_{1} \circlearrowleft M$. Hence there exists $h \in \cap F_{1}$ such that $h \in M$. Since $M$ is an $l$-ideal, $|h| \notin M$. By [7, I, 3.15 (iii) ], $f \wedge|h|=0$ implies that $f|h|=0$ and so $f|h| \in M$. By the primeness of $M, f \in M$ and thus $\bar{f}(M)=0$. It is easy to see that if $M \in Z_{2}$, then $\bar{f}(M)=1$. Moreover, we may assume that $0 \leqq \bar{f}(M) \leqq 1$ for all $M \in R(A)$ since $(f \vee 0) \wedge 1 \in A$. Thus $\bar{f}(M) \in R$ for all $M \in \mathfrak{M}(A)$ and so $\{M \in \mathfrak{M}(A) \mid \bar{f}(M)=0\}$ and $\{M \in \Re(A) \mid \bar{f}(M)=1\}$ are disjoint closed sets in $\Re(A)$ containing $Z_{1}$ and $Z_{2}$, respectively.

\section{REFERENCES}

1. F. W. Anderson, Approximation in systems of real-valued continuous functions, Trans. Amer. Math. Soc. 103 (1962), 249-271.

2. F. W. Anderson and R. L. Blair, Characterizations of certain lattices of functions, Pacific J. Math. 9 (1959), 335-364.

3. G. Birkhoff, Lattice theory, rev. ed., Amer. Math. Soc. Colloq. Publ., Vol. 25, Amer. Math. Soc., Providence, R. I., 1948.

4. R. L. Blair, Stone's topology for a binary relation, Duke Math. J. 226 (1955), 271-280. 
5. M. Henriksen and D. G. Johnson, On the structure of a class of archimedean luttice-ordered algebras, Fund. Math. 50 (1961), 73-94.

6. G. A. Jensen, Characterizations of the lattice of all real-valued continuous functions on a completely regular space, Dissertation, Purdue Univ., Lafayette, Ind., 1966.

7. D. G. Johnson, $A$ structure theory for a class of lattice-ordered rings, Acta Math. 104 (1960), 163-215.

8. M. Ward, The closure operators of a lattice, Ann. of Math. 43 (1942), 191-196.

UNIVERSITY OF FLORIDA AND

Mathematisch Centrum, Amsterdam 